\title{
Foreword
}

Magnetism is a field of condensed matter research whose scientific outcome has invaded our everyday life. It regularly provides new mechanisms that can be used to develop novel technologies, as well as fundamental concepts which enrich the human scientific knowledge. Amongst major current issues in magnetism are, for instance, the coupling of the magnetic degrees of freedom (spin, orbit) with others, like the charge or the lattice, in potential multifunctionnal materials, and the study of magnetism at different space and time scales, as a route towards applications in magnonics or spintronics.

The relevance of neutron scattering techniques for the study of magnetism does not have to be demonstrated anymore. This versatile microscopic probe has accompanied all the discoveries and emerging fields of research in magnetism, like the studies of magnetic monopoles, skyrmions or multiferroism.

This book gathers most of the written contributions to the lectures given in May 2012 in Seignosse (France), during the thematic school devoted to "Neutrons and Magnetism" organized by J. Robert, S. Petit (Laboratoire Léon Brillouin), B. Canals, V. Simonet (Institut Néel, CNRS et Université Grenoble Alpes), H. Mutka (Institut laue Langevin), under the patronage of the Société Française de la Neutronique.

The goal of the school was to propose courses to students, as well as confirmed researchers, eager to improve their expertise in neutron scattering. The school started with two lectures on the basics of magnetism and neutron scattering. They were followed by more advanced courses on magnetic diffraction, polarized neutrons, magnetic inelastic scattering, magnetic reflectometry and small angle scattering, and magnetic diffuse scattering in disordered systems. Two lectures on complementary techniques, Nuclear Magnetic Resonance and X-ray scattering techniques were also proposed. To complement the contents of these courses, several highlighted examples of nowadays research in magnetism, using neutron scattering, were developed: superconductivity, molecular magnetism, thin films and heterostructures, frustrated magnetism.

This thematic school also had another objective, related with the upcoming changes which will impact european neutron research with the announced closure (within 20 years) of most of the research neutron reactors, and the construction of the new European Spallation Source ESS in Lund (Sweden). The sophisticated techniques and tools which have been developed over decades for the use of the neutron beam provided by reactors will have to be adapted to pulsed sources. In this respect, this book can be considered as a state of the art of neutron scattering research in magnetism, and a starting point towards the generalized use of pulsed sources.

We would like to take the opportunity to thank all the speakers and participants, as well as our sponsors, for their help in organizing this school.

This is an Open Access article distributed under the terms of the Creative Commons Attribution License 4.0, which permits unrestricted use, distribution, and reproduction in any medium, provided the original work is properly cited. 


\section{Collection SFN}

\section{Préface}

Le magnétisme est un domaine de recherche en physique de la matière condensée qui a envahi notre vie quotidienne. Régulièrement, de nouveaux concepts voient le jour, enrichissent les connaissances scientifiques, et conduisent au développement de nouvelles technologies. Parmi les principales questions d'actualité en magnétisme on trouve par exemple l'étude du couplage des degrés de liberté magnétiques (spin, orbite) avec les degrés de liberté de charge ou de réseau, une propriété que l'on tente de mettre à profit dans les matériaux dits «multifonctionnels », particulièrement prometteurs en termes d'applications.

Dans ces thématiques de recherche qui relèvent du magnétisme, la pertinence des techniques de diffusion de neutrons n'est plus à démontrer. Cette sonde microscopique a accompagné toutes les découvertes récentes, comme la physique des monopôles magnétiques, celle des skyrmions ou encore celle des composés multiferroiques.

Ce livre rassemble la plupart des conférences données en mai 2012 à Seignosse (France), au cours de l'école thématique « neutrons et magnétisme » organisée par J. Robert, S. Petit Laboratoire Léon Brillouin), B. Canals, V. Simonet (Institut Néel, CNRS et Université Grenoble Alpes), H. Mutka (Institut Laue Langevin), sous le patronage de la Société Française de la neutronique. L'objectif de l'école était de proposer une série de cours aux étudiants, ainsi qu'à des chercheurs confirmés, désireux d'améliorer leur expertise dans le domaine de la diffusion des neutrons. L'école a débuté avec deux conférences, l'une reprenant les bases du magnétisme et l'autre celles de la diffusion des neutrons. Des cours plus avancés ont suivi, sur la diffraction magnétique, les techniques de neutrons polarisés, la diffusion inélastique magnétique, la réflectométrie magnétique, la diffusion aux petits angles et enfin la diffusion magnétique diffuse dans les systèmes désordonnés. Deux conférences sur des techniques « sœurs », résonance magnétique nucléaire et techniques de diffusion des rayons X ont également été proposées. Pour compléter le contenu de ces cours, plusieurs exemples emblématiques de la recherche actuelle en magnétisme, utilisant la diffusion de neutrons, ont été développés : la supraconductivité, le magnétisme moléculaire, l'étude des films minces et des hétérostructures, le magnétisme frustré.

Cette école thématique avait aussi un autre objectif, lié aux futurs changements qui auront un impact sur la recherche neutronique européenne, avec la fermeture annoncée (moins de 20 ans) de la plupart des réacteurs de recherche, et la construction de la nouvelle source européenne à spallation, l'ESS à Lund (Suède). Les techniques et les outils sophistiqués qui ont été développés au cours des dernières décennies pour l'utilisation des faisceaux de neutrons fournis par les réacteurs devront être adaptés à des sources pulsées. À cet égard, ce livre présente un état de l'art de la recherche scientifique utilisant la diffusion des neutrons en magnétisme, et un point de départ vers l'utilisation généralisée des sources pulsées. Nous aimerions profiter de l'occasion pour remercier tous les intervenants et participants, ainsi que nos sponsors pour leur aide dans l'organisation de cette école. 